\title{
Verifying $\chi$ Models of Industrial Systems with Spin
}

\author{
Nikola Trčka \\ Department of Mathematics and Computer Science, Eindhoven University of \\ Technology, P.O.Box 513, 5600 MB Eindhoven, The Netherlands
}

\begin{abstract}
The language $\chi$ has been developed for modeling of industrial systems. To obtain performance measures, its simulator has been successfully used in many industrial areas. However, for functional analysis, simulation is less applicable. Such analysis can be done in other environments. In this paper we present guidelines and techniques for translating $\chi$ specifications to PROMELA, the input language of the well known model checker SPIN. We highlight the differences between the two languages and show, in a step by step manner, how some of them can be resolved. We conclude by giving the translation scheme and apply it to a small industrial case study.
\end{abstract}

Key words: industrial systems, verification, the modeling language $\chi$, the model checker SPIN

\section{Introduction}

When designing industrial systems (machines, manufacturing lines, warehouses, factories, etc.) engineers make frequent use of simulation models to detect flaws and to optimize performance. The language $\chi$ has been developed especially for this purpose. It allows for the specifying of both discrete-event and continuous aspects of industrial systems and its simulator has been successfully applied to a large number of industrial cases, such as a car assembly line (NedCar [12]), a multi-product, multi-process water fab (Philips [7]), a brewery (Heineken), a fruit juice blending and packaging plant (Riedel [10]) and process industry plants ([1]). Simulation is a powerful technique for performance analysis, like calculating throughput and cycle time, but it is less suitable for functional analysis (sometimes called verification). It can for instance reveal that a system has a deadlock (it is unable to proceed) or that it sometimes has a certain behavior, but it cannot show that the system is deadlock-free nor that it always has a certain behavior. 
A most widely used verification technique today is model checking. This technique performs an exhaustive search of the state space checking if a certain property of the system holds. The property is represented as a formula of some temporal logic, a logic that allows us to say things like: if a machine is given input then it will eventually produce a correct output. There are many variants of these logics (consult e.g. [22]). They can be linear (reasoning is about a single sequence of states) or branching (reasoning involves several different branches starting from a state), action based (reasoning is about what action can be performed in a state) or state based (reasoning about the value of variables in a state), etc. Once the property is stated, model checking becomes a completely automated process.

To facilitate model checking, $\chi$ has to be turned into a formal method. Indeed, recently, a big part of the discrete-event subset of the language was formalized (the formalization is called $\chi_{\sigma}$ ); a structural operational semantics and a notion of equivalence were defined [5]. Moreover, a state space generator, a tool that generates all possible behaviors of the model, was built. Properties of the model, represented as alternation free $\mu$-calculus formulas (see [22] again), can then be verified by the model checker of the CADP toolset [9]. However, there are some problems concerning this approach. First, the current toolset is a prototype; to be used in real-world applications, it would need to be optimized. Second, $\chi$ has (the notion of states of) variables but their values are not kept in the state space and therefore cannot be used in the temporal logic formulas, i.e. model checking must be action based. To a large extent, formulas referring to values of variables can be verified with action based model checking but this always requires big changes of the model, e.g. adding of actions that notify that a variable has a certain value. In addition, not all of the temporal logic formulas have equivalents in the alternation free $\mu$-calculus [18]. The third (and probably the biggest) problem is that the (usually very large) state space must be generated completely before a verification within CADP can take place.

To solve some of these problems we consider verification of $\chi$ models in some other environments. Also, an extra motivation is that developers of $\chi$ are currently redesigning and extending the language [21] but have no intention to build any other tools besides a simulator. In particular, they do not intend to implement an optimized state space generator. Rather, their idea is to facilitate the verification of $\chi$ models by establishing a connection with other state-of-the-art verification tools and techniques.

The aim of this paper is to present techniques for translating general $\chi$ specifications to Promela, the input language of the popular model checker SPIN. Aspects in which $\chi$ and Promela differ from each other are given in a step by step manner and treated in detail. For each aspect, difficulties of translation are discussed, pitfalls and solutions presented and explained. We cover many 
important features of $\chi$ such as time, nested parallelism, urgency etc. that are usually present in models of industrial systems. We also (syntactically) define a subset of $\chi$ models that can be translated to PROMELA and present a translation scheme.

Our work can be seen as an extension of that presented in [4] and [3]. In [4], the authors present a translation of a $\chi$ model of a turntable machine to Promela and verify properties, like the absence of deadlock and no product loss, with SpIN. The focus is on the verification and not on the translation; general guidelines for translating arbitrary $\chi$ models to PROMELA are not provided. In [3], a more detailed model of the same machine is translated to Promela, $\mu$ CRL [2] and UpPaAl timed automata [19]. Even though this paper shows some techniques and difficulties of the translation to PrOMELA, its aim is to compare the different approaches for the functional analysis of $\chi$ models, and to a lesser extent on the aspect of translation.

Note that here we take an informal approach to the problem. In [20] we support a part of our translation with a formal correctness proof. There we define a notion of equivalence for (a slightly different version of) $\chi$, prove that it is a congruence and that it preserves validity of temporal logic formulas. Then, we identify a subset of $\chi$ that maps to PROMELA more straightforwardly and show how a bigger subset can be reduced, modulo the equivalence, to that form.

The structure of the paper is as follows. In Section 2 we give an introduction to $\chi$; its syntax and (informal) semantics. As an illustration we present a $\chi$ model of a small manufacturing line. In Section 3 we briefly introduce Promela, pointing out features that we need. Section 4 is the main section of this paper. There we explain how we deal with the aspects of $\chi$ that are uncommon to Promela: parallelism, scoping and complex data types, deadlock and immediate termination, guarded processes and time. For each feature we show what the problems of translating it to PROMELA are and how we can circumvent them. Then, we present a translatable subset of $\chi$ and a translation scheme. At the end of the section we illustrate the translation process by showing the PROMELA translation of the model of a manufacturing line introduced in Section 2. We also show the usefulness of our approach by proving a simple property of the line. In the last section we give some conclusions.

\subsubsection{Acknowledgements}

I thank Bas Luttik for useful comments. 


\section{The $\chi$ Language}

The redesign of the $\chi$ language (including features to model hybrid behavior) is still work in progress [21]. Since a large part of the discrete-event subset of the new version of $\chi$ can be expressed in $\chi_{\sigma}$, we take $\chi_{\sigma}$ as our starting point.

\subsection{Syntax and semantics of $\chi_{\sigma}$}

Here we give a short and informal introduction to the language. We refer to [5] for a complete syntax definition and a formal semantics of the language constructs.

\subsubsection{Data types}

The basic data types of $\chi_{\sigma}$ are booleans, natural, integer and rational numbers and (typeless) channels. Real numbers are not supported. Most of the usual constants, operators and relations are defined for every data type and can be used together with variables to build expressions. Furthermore, $\chi_{\sigma}$ provides a mechanism to build tuples, lists and sets from the basic types (but not channels).

\subsubsection{Time model}

The time domain in $\chi_{\sigma}$ is dense, i.e. timing is measured on a continuous time scale. The weak time determinism principle, sometimes called the time factorization property (time does not make a choice), is implicitly adopted. Maximal progress (a process can delay only if it cannot do anything else) can be enforced by an operator. Time additivity (if a process can delay first $t_{1}$ and then immediately $t_{2}$ time units then it can delay $t_{1}+t_{2}$ time units from the start) is not implemented. Delaying is enforced by the delay operator but some processes can also implicitly delay (see the next paragraph).

\subsubsection{Atomic processes}

The atomic processes of $\chi_{\sigma}$ are process constructors and they cannot be split into smaller $\chi_{\sigma}$ processes. They are:

(1) The empty process $(\varepsilon)$, the process whose only option is to terminate successfully. 
(2) The deadlock process $(\delta)$. It cannot execute any action, it cannot delay and it does not have the option to terminate successfully.

(3) The skip process. It performs the internal action $\tau$. It cannot delay.

(4) The delay process $(\Delta e)$. It delays any number of time units less or equal than the value of the expression $e$.

(5) The assignment process $(x:=e)$. It assigns the value of the expression $e$ to the variable $x$. It does not have the possibility to delay.

(6) The send process $(m ! e)$. It stores the value of the expression $e$ in the channel $m$ (which behaves as one-place buffer). It is able to delay arbitrarily long.

(7) The receive process $m ? x$. If the channel $m$ is not empty, then it assigns the value in $m$ to the variable $x$; otherwise it blocks. It is also delayable.

\subsubsection{Operators}

There are nine operators in $\chi_{\sigma}$. We present each one of them together with their (informal) semantics.

(1) The guard operator $(: \rightarrow)$. A process $b: \rightarrow p$ behaves as $p$ only if the value of the boolean expression (guard) $b$ is true, otherwise it deadlocks.

(2) The alternative composition operator (\). A process $p$ [ $q$ represents a non-deterministic choice between $p$ and $q$.

(3) The sequential composition operator (;). A process $p ; q$ behaves as $p$ followed by the process $q$.

(4) The repetition operator $(*)$. A process $p *$ behaves as $p$ zero or more times.

(5) The parallel operator $(\|)$. A process $p \| q$ executes $p$ and $q$ concurrently in an interleaved fashion, i.e. the actions of $p$ and $q$ are executed in arbitrary order. If one of the processes can execute the send action and the other one can execute the receive action on the same channel then they can also communicate; in other words $p \| q$ can also execute a communication action on this channel.

(6) The scope operator $(\llbracket[\mid])$. A process $\|s \mid p\|$ behaves as $p$ in a local state $s$. The state $s$ is used to define local variables and channels visible only to the process $p$. It is recursively defined as the empty state $\left(\lambda_{s}\right)$ or as $v: s^{\prime}$ where $s^{\prime}$ is a state and $v$ is a variable declaration $(x:$ type $[\mapsto c])$ or a channel declaration $(\sim m)$.

(7) The encapsulation operator $\left(\partial_{A}\right)$. A process $\partial_{A}(p)$ disables all actions of $p$ that occur in the parameter set $A$. Most of the time, the set $A$ contains only send and receive actions to enforce a rendez-vous (synchronous) communication.

(8) The maximal progress operator $(\pi)$. A process $\pi(p)$ behaves as $p$ only that it can delay only if $p$ can delay and $p$ cannot execute any action.

(9) The abstraction operator $\left(\tau_{I}\right)$. A process $\tau_{I}(p)$ 'hides' (renames to $\tau$ ) all actions of $p$ that occur in the parameter set $I$. 


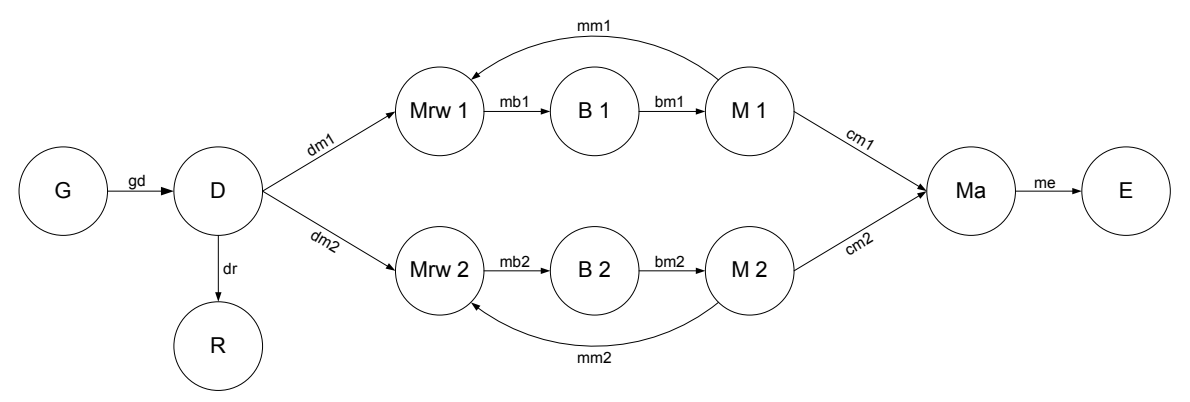

Fig. 1. Components of the manufacturing system

Note that $\chi_{\sigma}$ provides process definitions but this part of the language is not formalized so we do not consider it here.

\subsection{A manufacturing line in $\chi_{\sigma}$}

To give an impression of the language we give an example, a slight modification of the one given in [21]. Consider a manufacturing line that consists of a generator, a distributor, a rejector, two manufacturing cells, an assembly machine and an exit process. The generator $(G)$ generates products every 7 time units and delivers them to the distributor. The distributor $(D)$ waits 5 time units for one of the cells to be ready and then sends it a product. If in 5 time units none of the cells are ready the distributor sends a product to the rejector. Each manufacturing cell consists of two machines (Mrw and $M)$ and a 3 -place buffer in between $(B)$. Every product is processed by the cell twice. After processing, products are sent to the assembly machine $(M a)$ where they are processed further, combined and sent to the exit process $(E)$. Every machine takes 4 time units to perform its operation. The whole system is pictured in Fig. 1.

We now present a $\chi_{\sigma}$ model of the line:

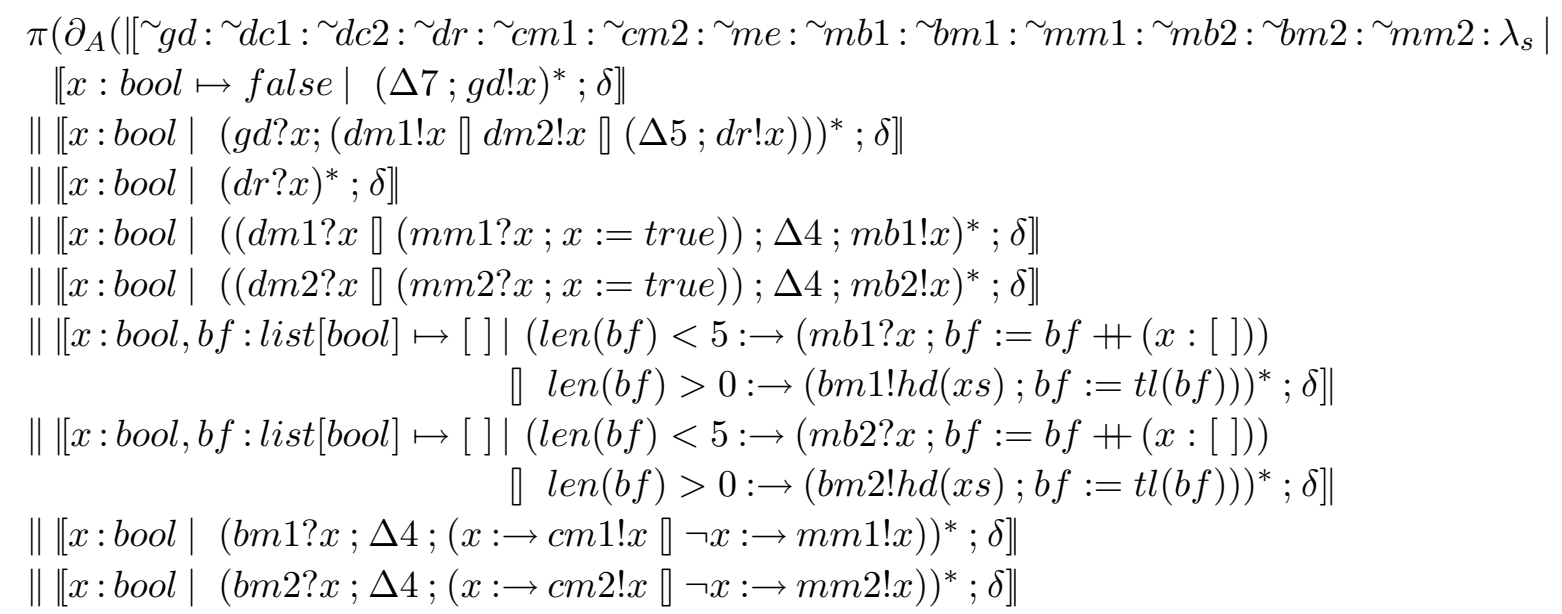


\|\|$x, y:$ bool $\mid((c m 1 ? x \| c m 2 ? y) ; \Delta 4 ; m e !(x \wedge y))^{*} ; \delta \|$

\|\|$\left[x:\right.$ bool $\left.\left.\left.\mid(m e ? x)^{*} ; \delta \|\right] \|\right)\right)$

Every parallel component represents a part of the system. The order is: $G$, $D, R, M r w_{1}, M r w_{2}, B_{1}, B_{2}, M_{1}, M_{2}, M a$ and $E$. A global scope is used for the declaration of channels that represent connection between parts. The set $A$ contains all send and receive actions. Variable $x$ models products. It is a boolean variable so that we can distinguish cases when the product was not processed by the cells yet ( $x$ is false) from when it is already processed once ( $x$ is true). Symbol [] denotes an empty list, $x:$ [ ] means that $x$ is added to the empty list, and the operator + concatenates two lists.

\section{Promela/Spin}

The full presentation of Promela, a very complex language, is beyond the scope of this paper. We give here only a brief overview mentioning only those parts of the language that we are interested in. For more information, see $[15,11,16]$ or consult SPIN's web page http://spinroot.com.

Promela's syntax is derived from $\mathrm{C}$ [17], with communication primitives from CSP [14] and control flow statements based on the guarded command language [8]. It has many language constructs similar to $\chi_{\sigma}$ constructs.

A common specification consists of global channel declarations, variable declarations and process declarations with possibly one special init process. Process declarations specify behavior, channel and variable declarations define the environment in which the processes run. Promela has a rather limited set of data types, only bool, byte, short, int (all with the unsigned possibility) and channels. It also provides a way to build records and arrays and to define C-like macros. Message channels are declared, for instance, as chan $\mathrm{m}$ $=[2]$ of $\{$ int $\}$ meaning that the channel is buffered and that it can store (at most) two values of (its field's) type integer. Channels can be of length 0 , i.e. unbuffered, to model synchronous communication. They can also have more than one field, not necessarily of the same type.

Every variable must be declared before use. The exception is the special dummy variable ', which is a predefined, global, write-only variable of type integer, used to store some input values that are of no importance later. It is an error to use or reference its value.

Process declarations are of this form:

proctype $\mathrm{P}$ (parameters) \{ 


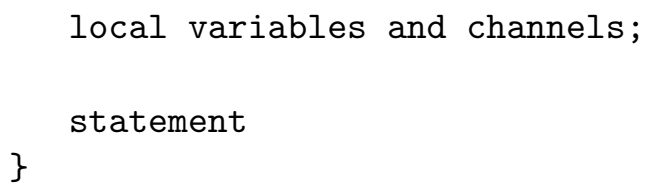

Local variables and channels specify the local state of the process and they are not visible to other processes. The same rules as for global variables apply here. Any expression is also a statement, executable precisely if it evaluates to a nonzero value. Assignments are also statements and have the usual semantics. The skip statement executes the action (1) and has no effect on variables. The send statement $\left(m ! e_{-} 1, \ldots, e \_n\right)$ sends a tuple of values of the expressions $e_{-} i$ to the channel $\mathrm{m}$. The receive statement $(\mathrm{m}$ ?E_1, . . E $\mathrm{E} n$ ) retrieves a message from the non-empty channel $m$, for every $E_{-} i$ that is a variable assigns a value of $e_{-} i$ to it and for every other $E_{-} j$ makes sure that its value matches the value of the $e_{-} j$. If the channel is buffered, a send is enabled if the buffer is not full; a receive is enabled if the buffer is non-empty. On an unbuffered channel, a send (receive) is enabled only if there is a corresponding receive (send) that can be executed simultaneously. There are also many variants of these statements.

There are several ways to combine statements. The alternative composition is defined by the selection statement:

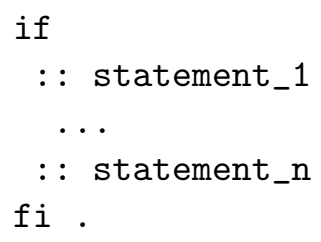

It nondeterministically selects among its options an executable statement and executes it. A selection blocks until there is at least one selectable option.

The repetition is achieved by the statement:

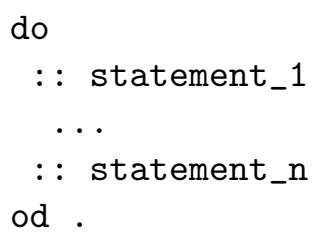

It is similar to the selection statement except that the choices are executed repeatedly, until control is explicitly transferred to outside the statement by a break or goto statement. The break statement terminates the innermost repetition statement in which it is executed and cannot be used outside a repetition.

Another way to combine statements is to use sequential composition denoted as $\mathrm{p}$; $\mathrm{q}$ or $\mathrm{b} \rightarrow \mathrm{p}$. The latter is usually used to emphasize that a process $\mathrm{p}$ is 
guarded by the conditional expression/statement $\mathrm{b}$.

The original version of PROMELA/SPIN is untimed but there is a discrete time extension, called DTPRomela/DTSPIN [6]. The idea is to divide time into slices and then frame actions into these slices. The time between actions is measured in ticks of a global digital clock. By having a variable $t$ declared as timer, setting its value to some expression that evaluates to a natural number (by doing set $(t, e)$ ) and waiting for $t$ to expire (by stating expire(t)) a process can be enforced to postpone its execution for $\mathrm{n}$ time slices (where $\mathrm{n}$ is the value of e). When DTSPIN executes the timeout action, all timers synchronize and time progresses to a next slice. This action is executed only if no other actions can be executed, meaning that maximal progress is implicit. Deadlock is recognized when timeout is about to happen and all timers are off (not set or already expired).

Promela provides two constructs, atomic\{stmt_1;...; stmt_n\} and $\mathrm{d}_{-}$step $\{$stmt_1; . . ; stmt_n\} that can be used to model indivisible events and to reduce a state space. Their purpose is to forbid the statements from inside to interleave with other statements in the specifications. The difference is that additionally d_step executes all statements as one (one state in the state space). These constructs are very useful but have limitations: statements other than the first may not block.

Once declared, every process can be started by the Promela process creation mechanism, the run statement. The special init process, if present, is automatically instantiated once, and is often used to prepare the true initial state of a system by initializing variables and running the appropriate processinstances. With the prefix active, a process definition is considered initially active and need not be started by the init process. Processes can be started with different parameters. Once started they execute in parallel with the interleaving semantics. This is the only way to achieve parallelism because there is no explicit parallel operator. Processes communicate with each other through global variables and channels.

Properties of a PROMELA model are expressed in a state-based linear temporal logic and verified by SPIN using the on-the-fly method of model checking (a property is checked while the state space is building, not after it is already built).

\section{Translating $\chi_{\sigma}$ to Promela}

First we introduce some mild assumptions about the $\chi$ processes we consider for translation. SPIN is a state based model checker and hiding of actions does 
not play a role so we assume that our models do not contain the $\tau_{I}$ operator. In addition, because the main form of communication in $\chi_{\sigma}$ is synchronous, we assume that the encapsulation operator $\left(\partial_{A}\right.$, with $A$ the set of all send and receive actions) is applied to our process. Since there is no explicit encapsulation in Promela, we do not allow $\partial_{A}$ to occur anywhere else. The last assumption concerns timing. Because maximal progress in SPIN is implicit, our process is prefixed also by the $\pi$ operator. This is the only place where $\pi$ is allowed (except for one special case, see Remark 1 below). To summarize, we consider only processes of the form $\pi \partial_{A}(p)$ where $p$ does not contain abstraction, encapsulation nor the maximal progress operator (experience shows that most $\chi_{\sigma}$ specifications of industrial systems are of this shape). From now on when we refer to the process we translate, we mean $p$.

\subsection{Techniques}

Translation of some $\chi_{\sigma}$ constructs, like assignments, the skip statement, sequential and alternative composition is straightforward since they have direct equivalents in Promela, but there are several important aspects in which $\chi_{\sigma}$ and Promela differ from each other. Translation of such features requires a detailed explanation. This will be presented in the following paragraphs.

\subsubsection{Parallelism}

As said before, process definitions from a PROMELA specification are implicitly executed in parallel and there is no (explicit) parallel operator. This means that our $\chi_{\sigma}$ process can have parallelism only on top level, i.e. in general, we must disallow nested parallel operators. In some cases however, there are techniques to deal with nested parallelism and we discuss them now.

Note that a sequential composition can be simulated by a parallel composition at the expense of introducing an extra synchronization variable. Thus process $(p \| q) ; r$ is equal to

$$
\|w: n a t \mapsto 0 \mid p ; w:=w+1\| q ; w:=w+1 \| w=2: \rightarrow r \rrbracket
$$

and similarly $p ;(q \| r)$ is equal to

$$
\| w: \text { bool } \mapsto \text { false } \mid p ; w:=\text { true }\|w: \rightarrow q\| w: \rightarrow r \| .
$$

This technique can easily be extended from two to an arbitrary number of parallel components.

If parts of a process that run in parallel do not communicate with each other, the parallel operator is just an interleaving operator. In both $\chi_{\sigma}$ and PROMELA 
interleaving of atomic processes can sometimes be achieved with one loop and a few additional guards (boolean variables). The idea is to associate one guard to each atomic process. If there is a choice between two atomic processes then they share the same guard. Only atomic processes available from the start have their guards initially set to true. When an atomic process is executed, its guard is put to false and the guard of the atomic process that comes next is assigned true. This is done in a loop that is exited when all the guards are false. Note that this does not work when there is a ${ }^{*}$ operator involved.

We illustrate the technique with an example. Suppose $a, b, c, d$ and $e$ are atomic processes. Then, process $a ; b \| c ;(d \llbracket e)$ is transformed to:

$$
\begin{aligned}
& \mid\left[b_{1}: \text { bool } \mapsto \text { true }, b_{2}: \text { bool } \mapsto \text { false }, b_{4}: \text { bool } \mapsto \text { false }, b_{3}: \text { bool } \mapsto \text { true } \mid\right. \\
& \left(b_{1}: \rightarrow a ; b_{1}:=\text { false } ; b_{2}:=\right.\text { true } \\
& \text { प] } b_{2}: \rightarrow b ; b_{2}:=\text { false } \\
& \text { I } b_{3}: \rightarrow c ; b_{3}:=\text { false } ; b_{4}:=\text { true } \\
& \text { ] } b_{4}: \rightarrow d ; b_{4}:=\text { false } \\
& \text { ] } b_{4}: \rightarrow e ; b_{4}:=\text { false } \\
& ) * ; \neg\left(b_{1} \wedge b_{2} \wedge b_{3} \wedge b_{4}\right): \rightarrow \varepsilon\right] \mid .
\end{aligned}
$$

Note that this solution introduces many additional assignments and therefore enlarges the state space of a process. When translating the example to Promela one can put a guarded command and the assignments following in a d_step statement.

So far, when dealing with nested parallelism, we worked mostly within $\chi_{\sigma}$, not considering features present only in PromelA. A good candidate for dealing with nested parallelism is Promela's process creation mechanism, i.e. the run statement. The process $p \| q$ is translated to

$$
\operatorname{atomic}\{\operatorname{run}(p) ; \operatorname{run}(q)\}
$$

and $p$ and $q$ become separate process definitions in Promela. However, this must be used with great care. If the parallel composition is in a choice context, e.g. $(p \| q) \rrbracket r$, then it cannot be translated as:

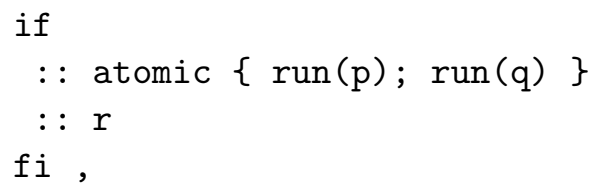

For in this Promela specification the choice does not depend on the executability of $p \| q$; the run statement is always executable. 


\subsubsection{Data Types}

From the set of basic data types the $\chi_{\sigma}$ specification can contain channels, booleans, natural numbers and integers. Rational numbers are not supported by Promela. Every undeclared variable must be translated to the dummy variable. Translation of bool, nat and int variable declarations is straightforward but translation of channel declarations requires more explanation. First, in Promela, forcing the communication on some channel to be handshake communication is automatically done if the channel is declared of zero length. Second, since all channels in $\chi_{\sigma}$ are typeless, in order to translate (declarations of) them to Promela we must first determine their type from the type of the receiving variable. This means, if we declare a channel as $\sim m$ and use it, for example, as $m ! 1$ and $m ? x$, where $x$ is declared as integer, then in PromelA $m$ should be declared as

$$
\text { chan } m=[0] \text { of }\{\text { int }\}
$$

and used as $\mathrm{m} ! 1$ and $\mathrm{m}$ ?x. Using channels to transport data of different types is therefore not allowed.

Complex data types can be implemented using PromelA's support for records, arrays and macro definitions. For example, a tuple of an integer and a boolean value (declared as tuple $[i n t, b o o l]$ in $\chi_{\sigma}$ ) is represented as

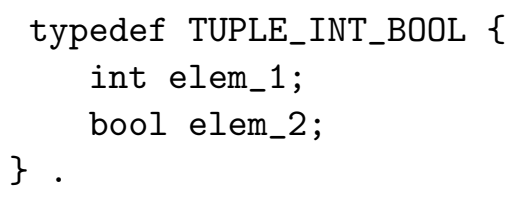

To model lists we can use buffered channels (a similar approach was taken in [13]). A list of length $n$ is defined as a tuple of a channel 1 of capacity $\mathrm{n}$ (the actual list) and a variable head that holds the first element of the list. Adding an item to a list is represented as sending it to a channel that represents the list. Transforming a list into its tail is done by receiving an element from this channel. To keep the head variable up-to-date, we use a predefined Promela function len that returns the length of a channel and a variant of the send statement $(m ?\langle x\rangle)$ that behaves as $\mathrm{m}$ ? $\mathrm{x}$ only that the message from the channel $\mathrm{m}$ is not erased upon executing. A list of $\mathrm{n}$ integers is represented as:

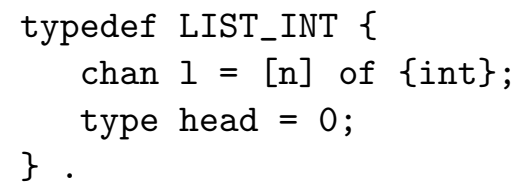

To make the usage of lists simpler and closer to $\chi_{\sigma}$ syntax we define four macros. They represent some usual functions on lists (note that $\chi_{\sigma}$ has more): $\operatorname{add}(\mathrm{x}, 1 \mathrm{st})$ adds $\mathrm{x}$ to the list 1 st, hd(1st) returns a first element of 1st, 
tail(lst) transforms the list lst into its tail and length(lst) gives the length of 1st.

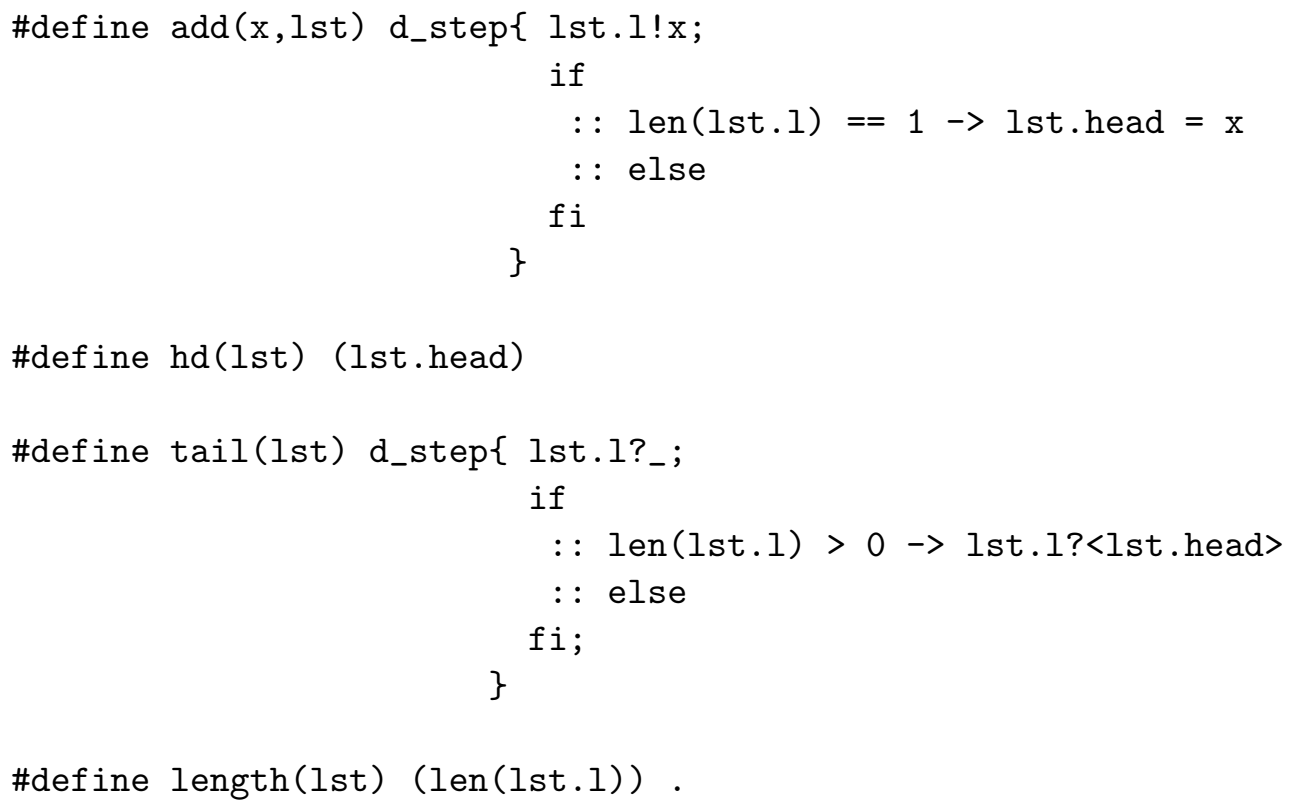

\subsubsection{Scoping}

In Promelathere are only two scope levels. Process local, in process declarations, and global, outside of them. It is not possible to introduce blocks with block-local variables inside the process declarations. This is not a serious limitation because for almost every process we can always find an equivalent one of the form $\left\|s\left|\left\|s_{1}\left|p_{1}\|\| \ldots\|\| s_{n}\right| p_{n}\right]\|\|\right.\right.$ where the $p_{i}$ 's do not contain the scope operator.

First note that $\| \lambda_{s}|p \rrbracket|$ is equivalent to $p$ and that $\left\|s_{1}\left|\| s_{2}\right| p\right]\|\|$ is equivalent to $\| \operatorname{set}\left(s_{1}, s_{2}\right) \mid p \rrbracket$ (where set is a function that adds variables from $\mathbf{s}_{-} 2$ to $\mathbf{s}_{-} 1$, overwriting those already present in $\mathbf{s}_{-} 1$ ). This allows us to eliminate scope when its declaration section is empty or when it is immediately nested.

Further, it is not hard to prove that, when $q$ does not contain free variables (a variable is free in $q$ if it is not used within a scope that declares it) that are declared in $s$, then $\|s \mid p\| \circ q$ is equivalent to $\|s \mid p \circ q\|$ for all $\circ \in\{;],, \|\}$. Similarly, $b: \rightarrow\|s \mid p\|$ is the same as $\| s|b: \rightarrow p \rrbracket|$ when $b$ does not contain variables also declared in $s$, and $p ; \| s \mid q \rrbracket$ is the same as $\| s \mid p ; q \rrbracket$ when the free variables of $p$ are not declared in $s$.

Elimination of a scope in the context of a repetition is more complicated. Note that the process $\| s \mid p]^{*}$ has different behavior than $\left\|s \mid p^{*}\right\|$. This is because $p$ in $\|s \mid p\|^{*}$, when it has finished executing, starts again in the 'fresh' state $s$ while $p$ in $\left\|s \mid p^{*}\right\|$ starts from a possibly changed state. A solution is to 
Table 1

Elimination of scopes

\begin{tabular}{|c|c|}
\hline$\| \lambda_{s} \mid p \rrbracket$ & $p$ \\
\hline$b: \rightarrow\|s \mid p\|$ & $\|s \mid b: \rightarrow p\|$ \\
\hline$\| s \mid p \rrbracket ; q$ & $\| s \mid p ; q \rrbracket$ \\
\hline$p ; \| s \mid q \rrbracket$ & $\| s \mid p ; q \rrbracket$ \\
\hline$\|s \mid p\| \rrbracket q$ & $\| s \mid p \rrbracket q \rrbracket$ \\
\hline$\|s \mid p\| \| q$ & $\|s \mid p\| q \|$ \\
\hline$\left\|s_{1}\left|\left\|s_{2} \mid p\right\|\|\|\right.\right.$ & $\| \operatorname{set}\left(s_{1}, s_{2}\right) \mid p \rrbracket$ \\
\hline$\|s \mid p\|^{*}$ & $\left.\| s \mid\left(p ; x_{1}:=c_{1} ; \ldots ; x_{n}:=c_{n}\right)^{*}\right]$ \\
\hline
\end{tabular}

make $p$ restore the old state when it is done. In other words, if $s$ is of the

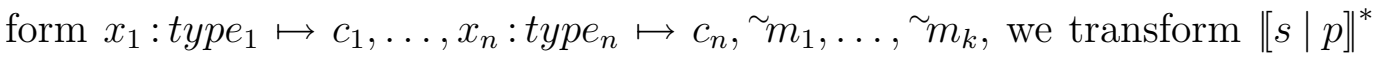
to $\left\|s \mid\left(p ; x_{1}:=c_{1} ; \ldots ; x_{n}:=c_{n}\right)^{*}\right\|$. If some of the $x_{i}$ 's are not initialized (i.e. the part $\mapsto c_{i}$ is missing) we simply omit $x_{i}:=c_{i}$.

The summary of all the transformations that (after adequately renaming variables) can be used for nested scopes elimination is given in Table 1.

\subsubsection{Deadlock and Immediate Termination}

The deadlock process $\delta$ has an equivalent in Promela, the boolean expression/statement false. The only difference is that, in PROMELA, deadlock is delayable until all timers are off. On the other hand, there is no notation to express (successful) immediate termination in Promela. Our process, therefore, cannot contain $\varepsilon$. To illustrate that $\varepsilon$ in general cannot simply be translated to the process skip we give an example. The $\chi_{\sigma}$ process skip $\llbracket \varepsilon ; \delta$ must always execute the skip action while the Promela statement

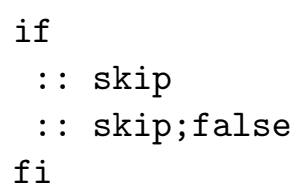

can also execute the second skip and deadlock afterwards.

In some cases it is possible to remove the $\varepsilon$ from a specification; using for instance that $\varepsilon ; p, p ; \varepsilon, p \| \varepsilon$ and $p \| \varepsilon$ are all equivalent to $p$ and that $(p \llbracket q) ; r$ is the same as $p ; r \llbracket q ; r$.

The problem with immediate termination is also hidden in the repetition. In $\chi_{\sigma}$, the behavior of the process $p^{*}$ is 'execute $p$ zero or more times' which 
means that it has an option to terminate immediately. Since $p^{*}$ is equivalent to $p ; p^{*} \llbracket \varepsilon$, we can think of it as it has a hidden $\varepsilon$. In PROMELA, the repetition operator do : : od cannot terminate without executing an action (break or goto). Therefore, it is not possible to translate $p^{*}$. However, process $p^{*} ; q$ (meaning $q$ or $p$ zero or more times and then $q$ ), if $q$ does not terminate immediately, has an equivalent in Promela:

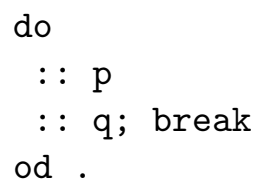

Hence, in the process we can translate, every occurrence of * must be followed by the sequential operator.

Note that an infinite repetition of a process $p$ in $\chi_{\sigma}$ is expressed as $p^{*} ; \delta$. Its translation to PROMELA is

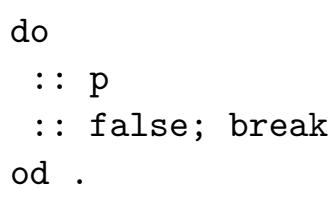

Because the expression/statement false is never executable the latter statement is equivalent to

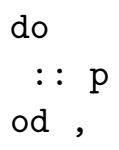

which is a usual way of presenting an infinite behavior in Promela.

Remark 1 Although break and goto statements should not be considered as actions but only as control flow mechanisms,

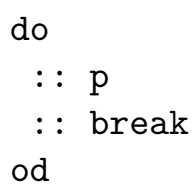

(similarly with goto) is not a right translation of the process $p^{*}$. This is because the break statement is still involved in a choice. The previous example is an illustration of this. If $p^{*} ; \delta$ were translated as

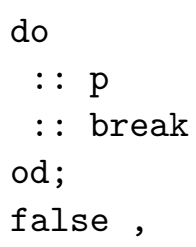


SPIN could always decide to change the control to the outside of the loop and deadlock.

\subsubsection{Delays}

DTPRomela is a discrete time extension so we require delays to be natural numbers. For rational ones there is always a number we can multiply all of them by, and obtain natural delays of the same ratio. The $\Delta e$ statement is translated to the DTPRomela statement expire(t), where $t$ is of type timer and is previously set to the value of $e$. For each $\Delta$ statement a new timer should be introduced. In cases where $\Delta e$ is not involved in a choice, set $(t, e)$ can be present immediately before the expire(t) (there is a Promela macro delay $(t, e)$ defined as set $(t, e)$; expire $(t)$ that can be used instead). However, when there is a choice of $\Delta e$ and another process we have to be more careful. If, for example, we translate $\Delta e \llbracket p$ as

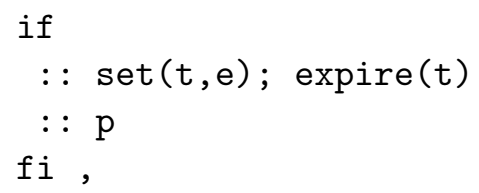

then, because set $(t, e)$ is always executable, SpIN can choose to execute it. For example, if $\mathrm{p}$ can do a send or receive action then we lose an option to communicate which contradicts the fact that send and receive processes are delayable and that alternatives delay together. Also, if $\mathrm{p}$ can do an assignment action, SpIN should not execute set $(t, e)$ because of the maximal progress. Similar problem appears in processes of the form $\Delta e^{*} ; p$.

To prevent time from making a choice set $(t, e)$ must be moved before the alternative composition (or repetition in the latter case). This is enough to assure the right behavior since expire( $t)$ is a boolean expression/statement that is blocked until (the value of ) e time slices later. Therefore, the right translation of $\Delta e \rrbracket p$ is:

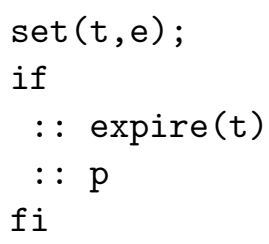

and similarly for do : : od statement.

Note that delaying zero time units in $\chi_{\sigma}$ is equivalent to $\varepsilon$, while in PromelA it is equivalent to skip, i.e. it does an action. This causes similar problems as the translation of $\varepsilon$ to skip would. For example, the process $\Delta 2 ; p \square \Delta 2 ; q$ 
waits two time units and then behaves as $p[q$. If we translate this process as

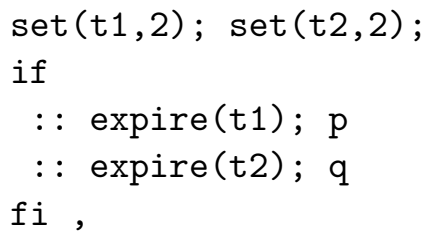

then after two clock ticks the values of $t 1$ and $t 2$ become 0 , the statements expire(t1) and expire(t2) become executable, and choice is made regardless of the executability of $\mathrm{p}$ and $\mathrm{q}$. Therefore, we can only translate $\chi_{\sigma}$ specifications in which all delay statements are immediately followed by the skip statement.

Remark 2 We have said already that send and receive processes can implicitly delay and that we assume that the maximal progress operator is only on top of the specification. However, when send or receive should be urgent, i.e. not delayable, we need to use $\pi(m ! e)$ instead of just m!e (same for receive). We can achieve the same in PROMELA with:

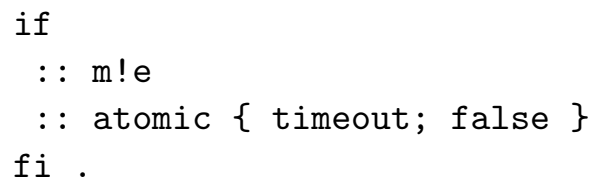

This statement says that the send is available but the passage of time leads to an immediate deadlock.

\subsubsection{Guards}

Statements of type $b: \rightarrow p$, in general cannot be just translated as $\mathrm{b} \rightarrow \mathrm{p}$. This is because in PROMELA operator $\rightarrow$ is equivalent to the sequential operator and the boolean expression $\mathrm{b}$ is also a statement. This means, if the value of $\mathrm{b}$ is true, SPIN will execute the action (1) (e.g. it will pass the guard) even though process $\mathrm{p}$ cannot execute anything. This is different from $\chi_{\sigma}$ which looks for both $b$ to be true and for $p$ to be executable before taking the step. For example, in $\chi_{\sigma}$, the process (true $: \rightarrow \delta$ [true $: \rightarrow$ skip) will execute skip because $\delta$ is never executable. In Promela however, process

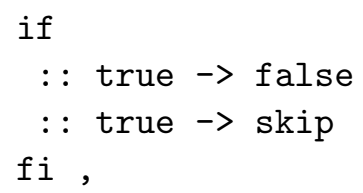

since it does not look 'behind' guards, can pick the first true, execute it and deadlock afterwards. Thus, the Promela statement $b \rightarrow p$ actually corre- 
sponds to the process $(b: \rightarrow s k i p) ; p$ in $\chi_{\sigma}$.

In the special case when $p$ is an atomic process it is always possible to translate process $b: \rightarrow p$. A guarded skip is translated to a PROMELA expression/statement $\mathrm{b}$. Guarded delays $(b: \rightarrow \Delta e)$ are simply translated as $\mathrm{b} \& \&$ expire( $t$ ) provided that $t$ is previously set to the value of e. A guarded assignment, $b: \rightarrow x:=e$, we translate as $d_{-} \operatorname{step}\{\mathrm{b} ; \mathrm{x}=\mathrm{e}\}$. With the d_step operator we force the statement to be executed as one action, like in $\chi_{\sigma}$. If the value of $b$ is false the statement is blocked, and if it is true, since an assignment is always executable, the statement will execute only one action.

In order to translate guarded send/receive actions we must apply a different trick because these actions can block. For a channel that has send/receive actions involved in guarded statements we first change the declaration by adding another field argument to it, one of type integer. We need the extra argument to synchronize on guards and we translate $b: \rightarrow m ! e$ to $\mathrm{m} ! \mathrm{e}, \mathrm{b}$ and $B: \rightarrow m ? x$ to $\mathrm{m}$ ? $\mathrm{x}$, eval (2-B). We use 2-B instead of just $\mathrm{B}$ because we want to avoid the communication between a guarded send and a guarded receive to happen when both guards evaluate to false $(2-B=b$ is equivalent to $B=1$ and $b=1)$. The eval function is used to force the evaluation of the expression 2-B. SpIN does not do this automatically in receive statements because the expression can be a variable in which case it should not serve as a match but instead it would be assigned the incoming value. If a communication action, for example $m ? x$, is not used in the guarded context but its counterpart send is, then it should be translated to $\mathrm{m}$ ? $\mathrm{x}, 1$. This goes similarly for $m$ !e when a corresponding receive is guarded.

Remark 3 There is another possibility to translate guarded send and receive statements on unbuffered channels (see page 398 in [15]). Instead of adding a boolean argument to a channel declaration we could declare an array of channels of size 3 and then translate $b: \rightarrow m$ !e to $\mathrm{m}[\mathrm{b}]$ ! e and $B: \rightarrow m ? x$ to $\mathrm{m}[2-\mathrm{B}]$ ? $\mathrm{x}$. Since $\mathrm{b}, \mathrm{B} \in\{0,1\}$ we force all the communication to happen on channels with index 1 . As an array index, expression 2-B evaluates automatically so there is no need for the eval function.

Like in the case of the scope operator, the restriction to have only guarded atomic processes is not so serious since most processes have equivalents in that form. Transformations that simplify guards in the context of other guards, alternative and sequential composition, and repetition are shown in Table 2 (how guarded scopes are simplified we have shown in the paragraph on scoping). Note that the solution for a guarded repetition introduces $\varepsilon$, but if $p^{*}$ is followed by the sequential operator the $\varepsilon$ is redundant.

In general, $b: \rightarrow(p \| q)$ is not equivalent to $(b: \rightarrow p) \|(b: \rightarrow q)$. This is because when the value of $b$ is true, after executing an action (for example from $p$ ) 
Table 2

Simplification of guards

\begin{tabular}{|l|l|}
\hline$b_{1}: \rightarrow b_{2}: \rightarrow p$ & $\left(b_{1} \wedge b_{2}\right): \rightarrow p$ \\
\hline$b: \rightarrow(p \llbracket q)$ & $(b: \rightarrow p) \llbracket(b: \rightarrow q)$ \\
\hline$b: \rightarrow(p ; q)$ & $(b: \rightarrow p) ; q$ \\
\hline$b: \rightarrow p^{*}$ & $(b: \rightarrow p) ; p^{*} \square b: \rightarrow \varepsilon$ \\
\hline
\end{tabular}

process $b: \rightarrow(p \| q)$ proceeds as $p^{\prime} \| q$ and process $(b: \rightarrow p) \|(b: \rightarrow q)$ as $p^{\prime} \|(b: \rightarrow q)$ and the action might have changed the value of $b$ to false. Only when $p$ and $q$ do not change the value of $b$, e.g. when they do not contain atomic processes that influence variables present in $b$, we can distribute the guard over the parallel operator.

\subsection{Translation scheme}

In this section we present the subset of $\chi_{\sigma}$ models that can be translated to Promela code and present the translation scheme. The subset is generated by the following grammar:

$$
\begin{aligned}
& A::=\text { skip }|x:=e| m ! e|m ? x| \pi(m ! e)|\pi(m ? x)| \Delta e ; \text { skip } \\
& B P::=\delta\left|b: \rightarrow B P_{1}\right| B P_{1} ; B P_{2}\left|B P_{1}\right| B P_{2}\left|B P_{1}^{*} ; B P_{2}\right|\left\|S \mid B P_{1}\right\| \\
& P::=\pi\left(\partial_{A}\left(\left\|S \mid B P_{1}\right\| \ldots\left\|B P_{n}\right\|\right)\right), n \geq 1
\end{aligned}
$$

Here $S$ is a state, of the same form as in the explanation of the scope operator of $\chi_{\sigma}$ but with the restricted number of data types that variables can be declared of. The symbol $e$ represents any expression and the symbol $b$ represents a boolean expression.

The grammar above allows nested scopes and arbitrary guarded processes. These statements do not have direct translations to Promela code, but, to eliminate them, we can perform the transformations from Tables 2 and 1 . In this (preprocessing) step we can also introduce the new symbol $\star$ that abbreviates the combination *; . The reduced processes are generated by the following grammar ( $A$ stays the same):

$$
\begin{aligned}
& B P::=\delta|b: \rightarrow A| B P_{1} ; B P_{2}\left|B P_{1} \llbracket B P_{2}\right| B P_{1} \star B P_{2} \\
& P::=\pi\left(\partial _ { A } \left(\left\|S \left|\left\|S_{1}\left|B P_{1} \rrbracket\|\ldots\|\left\|S_{n} \mid B P_{n}\right\| \|\right)\right), n \geq 1\right.\right.\right.\right.
\end{aligned}
$$

We now present the translation scheme:

$$
\delta \longmapsto \text { false }
$$




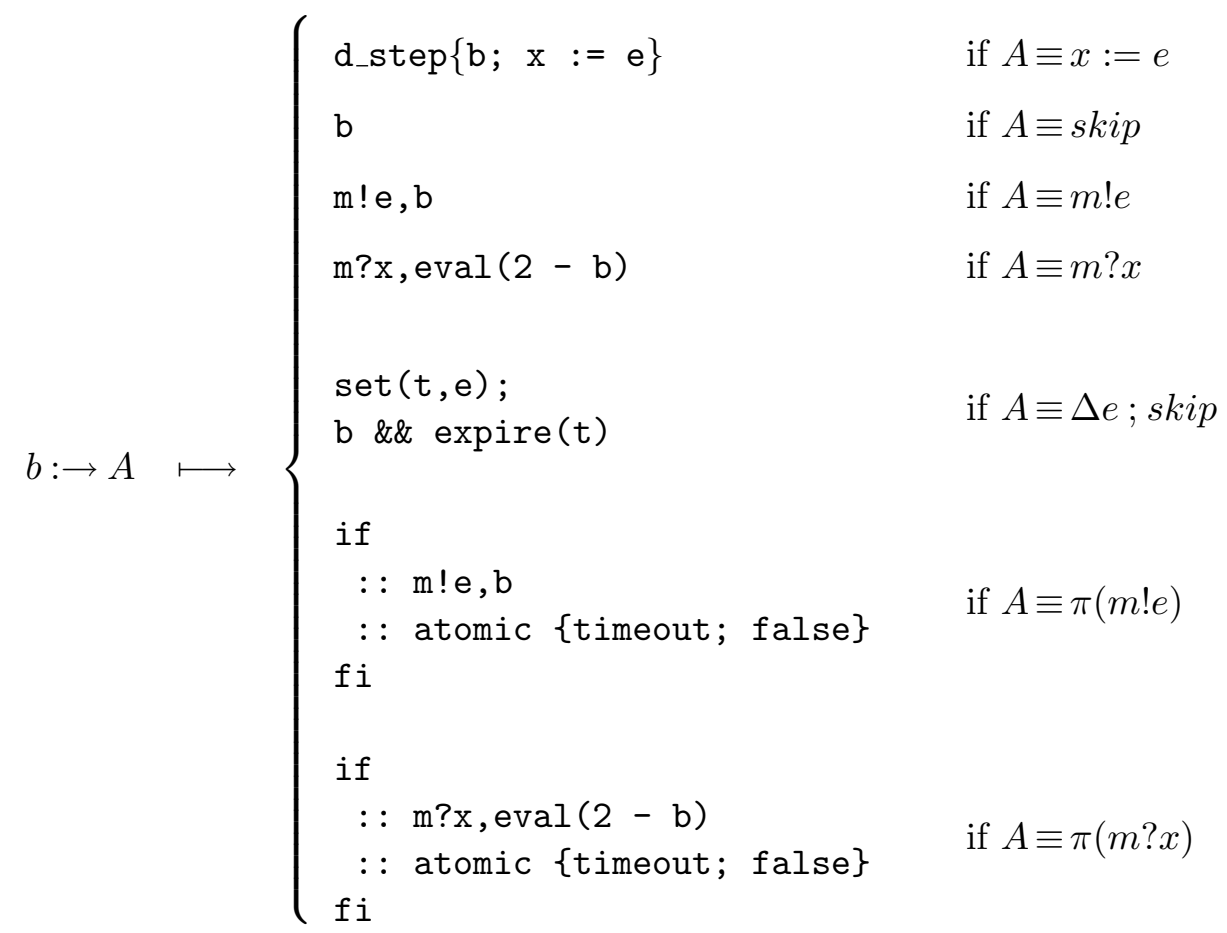

$B P_{1} ; B P_{2} \quad \longmapsto \quad \mathrm{BP} 1 ; \mathrm{BP} 2$

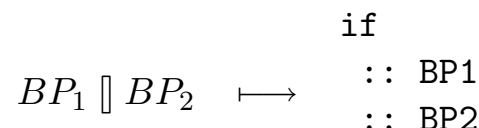

$$
\begin{aligned}
& \text { fi } \\
& \text { do }
\end{aligned}
$$

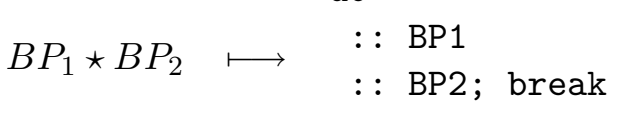

$$
\begin{aligned}
& \text { od } \\
& \mathrm{S} \text {; } \\
& \text { active proctype } \mathrm{P} 1() \text { \{ } \\
& \text { S_1 } \\
& \text { BP_1 } \\
& P \longmapsto \quad \text { ? } \\
& \text { active proctype } \operatorname{Pn}()\{ \\
& \text { S_n } \\
& \text { BP_n } \\
& \text { \} }
\end{aligned}
$$




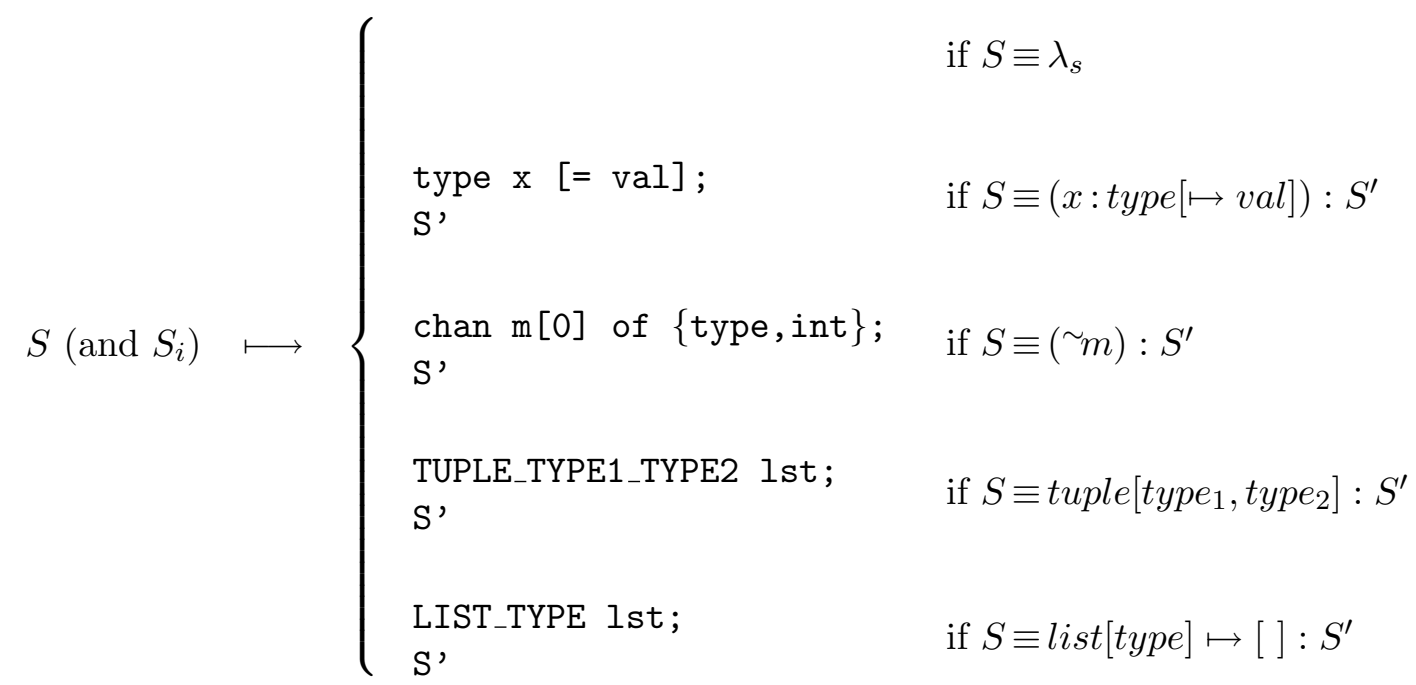

Note that, to correctly translate delays, a postprocessing step that declares and renames timers and that moves set functions to outside of if : : fi and do : : od statements, should be performed.

\subsection{Manufacturing line in PROMELA}

To illustrate our techniques and translation scheme we present the PromelA translation of the manufacturing line model presented in Section 2.2.

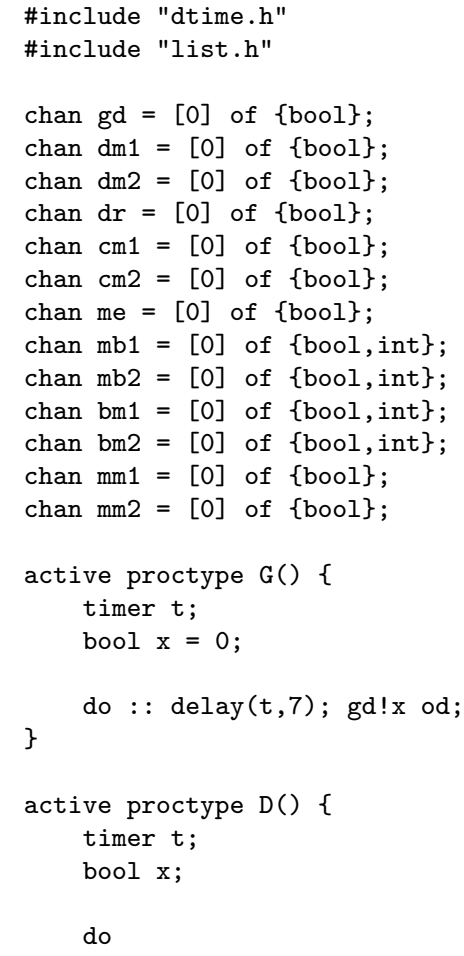

do 
also that sometimes, what seems to be an obvious translation, can have very different behavior.

Some constructions that we cannot translate, like e.g. $\varepsilon$ and $p^{*}[q$, are very rare in models of industrial systems. On the other hand, terms like $(p \| q)^{*}$ are quite common in such models. Nested parallelism is a major problem that we cannot (yet) deal with in a satisfactory way. This is a subject for further investigations.

We were able to syntactically define a translatable subset and we presented a translation scheme. We also defined the phases of a translation process. Based on this we are developing an automatic translator from $\chi$ to PROMELA.

Together, the simulator of $\chi$ and a tool that translates $\chi$ models into PromeLA will constitute an effective environment in which in which performance analysis and functional analysis of industrial systems are combined.

\section{References}

[1] D. A. van Beek, A. van der Ham, and J.E. Rooda. Modelling and control of process industry batch production systems. In 15th Triennial World Congress of the International Federation of Automatic Control, Barcelona, Spain, 2002.

[2] S. Blom, W. Fokkink, J.F. Groote, I. van Langevelde, B. Lisser, and J.C. van de Pol. $\mu$ CRL: A toolset for analysing algebraic specifications. In Proceedings of CAV2001, LNCS 2102, pages 250-254, 2001.

[3] E. Bortnik, N. Trčka, A.J. Wijs, S.P. Luttik, J.M. van de Mortel-Fronczak, J.C.M. Baeten, W.J. Fokkink, and J.E. Rooda. Analyzing a $\chi$ model of a turntable system using SpIn, CADP and UpPAAL. Technical Report CS-04/23, Eindhoven University of Technology, 2004. To appear in Journal Of Logic and Algebraic Programming.

[4] V. Bos and J.J.T. Kleijn. Automatic verification of a manufacturing system. Robotics and Computer Integrated Manufacturing, 17:185-198, 2001.

[5] V. Bos and J.J.T. Klein. Formal Specification and Analysis of Industrial Systems. PhD thesis, Eindhoven University of Technology, 2002.

[6] D. Bošnački. Enhancing State Space Reduction Techniques for Model Checking. $\mathrm{PhD}$ thesis, Eindhoven University of Technology, 2001.

[7] E. J. J. van Campen. Design of a Multi-Process Multi-Product Wafer Fab. PhD thesis, Eindhoven University of Technology, 2000.

[8] E. W. Dijkstra. A Discipline of Programming. Prentice-Hall, 1976. 
[9] J.-C. Fernandez, H. Garavel, A. Kerbrat, L. Mounier, R. Mateescu, and M. Sighireanu. CADP - a protocol validation and verification toolbox. In Proceedings 8th of CAV'96, LNCS 1102, pages 437-440, 1996.

[10] J.J.H. Fey. Design of a Fruit Juice Blending and Packaging Plant. PhD thesis, Eindhoven University of Technology, 2000.

[11] R. Gerth. Concise Promela reference. Obtainable from: http://spinroot. com/spin/Man/Quick.html.

[12] J. A. Govaarts. Efficiency in a lean assembly line: a case study at NedCar born. Master Thesis, October, 1997.

[13] Klaus Havelund, Mike Lowry, and John Penix. Formal analysis of a space-craft controller using SPIN. IEEE Trans. Softw. Eng., 27(8):749-765, 2001.

[14] C. A. R. Hoare. Communicating Sequential Processes. Prentice Hall, 1985.

[15] G. J. Holzmann. The SPIN model checker. Addison-Wesley, 2003.

[16] G.J. Holzmann. The model checker SPIN. IEEE Trans. on Software Engineering, 23(5):279-295, May 1997. Special issue on Formal Methods in Software Practice.

[17] B. W. Kernighan and D. M. Ritchie. The C Programming Language, Second Edition. Prentice-Hall, 1988.

[18] O. Kupferman and M.Y. Vardi. Freedom, weakness, and determinism: From linear-time to branching-time. In 13th IEEE Symposium on Logic in Computer Science, Indianapolis, Indiana, USA, 1998.

[19] K.G. Larsen, P. Pettersson, and W. Yi. UpPaAL in a nutshell. Int. Journal on Software Tools for Technology Transfer, 1(1-2):134-152, 1997.

[20] B. Luttik and N. Trčka. Stuttering congruence for $\chi$. Technical Report CS05/13, Eindhoven University of Technology, 2005.

[21] R.R.H. Schiffelers, D.A. van Beek, K.L. Man, M.A. Reniers, and J.E. Rooda. Syntax and consistent equation semantics of hybrid Chi. Technical Report CS04/37, Eindhoven University of Technology, 2004.

[22] C. Stirling. Modal and Temporal Properties of Processes. Springer, 2001. 